\title{
ONTIC STRUCTURAL REALISM AND MODALITY
}

\author{
NORA BERENSTAIN AND JAMES LADYMAN
}

\section{INTRODUCTION}

Ontic structural realism (OSR), as originally developed by Steven French and James Ladyman, incorporates an explicit commitment to the claims that the world has an objective modal structure and that this structure is represented by the theoretical structure of our best scientific theories. OSR attributes a rich structure of natural necessity to the world, and this distinguishes it from Bas van Fraassen's structural empiricism as well as from John Worrall's epistemic structural realism, both of which reject natural necessity. Ladyman and Ross [12], Ladyman [13] and Ross [21] extend OSR from physics to the special sciences and invoke the notion of the objective modal structure of reality in their accounts of novel prediction, causation, and real-patterns ontology. One may think about the modal structure discovered by science primarily in terms of causes or in terms of laws of nature. Either way, it is reasonable to question the extent to which the commitment to objective modality is essential to the defense of OSR and whether enough has been done by the above authors to justify its inclusion in their avowedly naturalistic metaphysics.

It is instructive in this context to consider whether ordinary scientific realism requires a commitment to natural necessity. While there has been surprisingly little direct discussion of this question in the realist literature, it is an implicit issue throughout the debate about scientific realism and an explicit one in debates about causation, explanation, and laws of nature. David Armstrong, Alexander Bird, Nancy Cartwright, Anjan Chakravartty, Brian Ellis, Ron Giere, and Ernan McMullin are all realists and anti-Humeans. However, Barry Loewer, David Papineau, and Stathis Psillos argue for the combination of scientific realism and Humeanism. On the antirealist side, it is natural to associate the denial of scientific realism with empiricism and the rejection of necessary connections in nature. This view motivated the logical positivists and logical empiricists in their critiques of realism, and it was also the view held by their intellectual ancestors. More recently, van Fraassen has repeatedly argued that scientific realism requires modal metaphysics and is for this very reason to be rejected by empiricists. (Of course, the above-mentioned Humean scientific realists will deny both of his claims.) On the other hand, it is clearly possible to deny important components of scientific realism and still be a realist about modality in other contexts. Non-naturalists, for example, might privilege agency and the manifest image in such a way as to be anti-Humean in their metaphysics while also making the repudiation of the scientific image central to their philosophy.

In this paper, we argue that not only is a reliance on objective modality integral to arguments for scientific realism but so too is a commitment to natural necessity. We also 
argue that the most popular and viable form of Humean scientific realism faces serious difficulties and therefore that the scientific realist must embrace anti-Humeanism about laws and causation. Before delving into these arguments, however, the notion of objective modality must be clarified. In accordance with methodological naturalism, we formulate our notion of objective modality not simply in the abstract but with reference to actual science. Consider, for instance, the cosmic speed limit of the speed of light. That material bodies do not in fact exceed $c$ is not what is usually claimed; rather it is said that material bodies cannot go faster than light. It is also generally pointed out that it would take an infinite force to accelerate an ordinary massive object to the speed of light, because in any frame in which the body were traveling anywhere close to light speed it would be unimaginably heavy. The question is whether modal claims such as these have their truthvalues independently of our current theories and models. If they do, then the modal facts are objective in the sense that they do not depend on our doxastic or epistemic states. If we think in terms of laws, then the question is whether the laws of nature are what they are because of how the world is independently of the way we as conscious subjects describe it. The matter may also be put in terms of causation. In the idiom of contemporary metaphysics, we may ask whether there are genuine causal powers.

Of course, it is possible to deny the existence of natural necessity while allowing that the modal language of science captures the way the world is independently of us. For example, it is possible to give a reductive account of modal facts that does not reduce them to anything that is dependent on us. This means that it is not correct to contrast Humeanism with the acceptance of objective modality. Rather, Humeanism is opposed to irreducible objective modality, or any kind of primitive natural, nomological, or causal necessity. ${ }^{1}$ Humeans deny that there is natural necessity but may nonetheless offer an account of objective modality. The sophisticated Humean may contend that the realist arguments for natural necessity depend only on there being objective modality, for which natural necessity is sufficient but not necessary. Hence, he will argue, Humeans can have the best of both worlds and avail themselves of the explanatory and dialectic advantages of scientific realism without incurring the metaphysical cost. We deny the tenability of this position.

In the next section, we review scientific realism and argue that realists are prima facie committed to objective modality and that the main argument for realism deepens that commitment. In section 3, the most defensible form of Humeanism in the contemporary literature is introduced and criticized as being incompatible with the spirit of scientific realism. Section 4 is a brief conclusion.

\section{Scientific Realism and Objective Modality}

Historically, there has been a connection between those who have criticized scientific realism and those who have opposed metaphysics of all kinds, particularly modal metaphysics. Scientific realists, on the other hand, are often distinguished from one another by

\footnotetext{
${ }^{1}$ We take natural necessity to be that to which one is committed when one accepts realism about laws of nature as something over and above regularities among actual events.
} 
their particular ontologies of causes, laws, and properties. However, if we define scientific realism simply as the view that our best scientific theories commit us to the existence of certain unobservable entities, then the position need not entail the existence of natural necessity. Rather, the modal implications of scientific realism come from the arguments in favor of the position and from the way its advocates articulate their view of science. In any case, it is not yet clear what it means to say that scientific realism is committed to the existence of natural necessity or simply to objective modality of some sort, as there is no widespread agreement on what counts as scientific realism. Let us review some of the relevant controversial propositions that are taken to characterize the view:

(1) Many or at least some of our best scientific theories and laws are true (or approximately true) claims about the world.

(2) Many or at least some of the theoretically described unobservable entities that we talk about exist.

(3) Many or at least some of the theoretical terms in our best scientific theories genuinely refer to unobservable entities.

(4) The aim of science is truth, not mere empirical adequacy.

(5) The best explanation of the success of science is that our theories are correctly describing the unobservable causes of what we observe.

1 implies a commitment to laws but not necessarily to causation. It is important to note that philosophers of science are divided over whether causation is a fundamental feature of reality, following Russell's famous claim that it does not feature in advanced sciences such as fundamental physics. In any case, 1 does not make it explicit whether laws are to be understood in genuinely modal terms or just as generalizations over regularities. The Stanford school of philosophers of science, notably Nancy Cartwright and Ian Hacking, developed entity realism and assert 2 but deny 1 . Obviously, they are not ontologically committed to objective modality in virtue of some commitment to laws of nature. However, they do invoke a non-Humean notion of causation, and indeed Cartwright [3] is a sustained attack on Humeanism about causation, which proposes to replace the view with a metaphysics of causal capacities. Here, the commitment to objective modality stems from the implicitly modal nature of the notion of capacity.

The reference of theoretical terms has been much discussed both in the abstract as well as in concrete scientific cases. The causal theory of reference was central to Hilary Putnam's post-positivist development of scientific realism and is still central to many scientific realist responses to the pessimistic meta-induction, such as Hardin and Rosenberg [9]. This is an example of how objective modality may be a commitment incurred by some realists not in virtue of their realism but in virtue of their arguments for it (see $\S 2.2$ below). ${ }^{2}$ While the distinction between truth and empirical adequacy may seem modally neutral, note that it is often spelled out in terms of the distinction between explaining the phenomena

\footnotetext{
${ }^{2}$ Psillos [19] develops a hybrid causal descriptivist theory of reference and applies it to various cases of abandoned theoretical terms. While he is officially a Humean, there is a question as to whether the causal theory of reference is viable if one denies that causation may be a singular relation between language users and events.
} 
and merely describing them. Note also that it may be argued that even the notion of empirical adequacy is modally committing in so far as it pertains to a description of the observable phenomena and not merely the actually observed phenomena. ${ }^{3} 5$ is one form of the argument for scientific realism based on inference to the best explanation, where this is construed as a causal explanation of the observable in terms of the unobservable.

2.1. Prima Facie Considerations: Modal Notions in Science. In rough terms, realists are those who take the theoretical claims of science at face value and who regard scientific theory choice as the final word in the epistemology of particular scientific theories and statements. Realism requires rejecting both revisionary philosophical versions of scientific theories and philosophical arguments for any skepticism that goes beyond the scientific community's healthy epistemic caution, exemplified by its insistence on empirical testing. If theoretical claims about electrons are to be taken literally as referring to unobservable entities bearing certain properties, then so too should claims about laws, causes, and other modalities. The following brief tour through modal notions in science is intended to establish that the skeptic about objective modality in general and natural necessity in particular faces the burden of proof and must offer some positive account that makes sense of the ubiquity of modal notions in science.

\section{1: Laws}

The pursuit of laws of nature is so central to the practice of science that establishing a body of laws concerning some domain is often considered to be a necessary condition for the formation of a science. Consequently, it has been argued that economics and other social sciences are not properly scientific because of the paucity of empirically confirmed laws in their domains. Both critics and advocates of social science have claimed that it is at least fundamentally different from natural science because of its deficit of laws. The status of physics, on the other hand, is due in large part to its success in reducing the complexity of nature to the operation of a few fundamental laws, beginning with Newton's three laws of mechanics and law of universal gravitation and culminating in the symmetry principles and conservation laws catalogued in the last century. Popular and semi-popular debates among physicists about the origins of the universe and the so-called 'fine-tuning problem' make reference to strong distinctions between contingent facts like the values of free parameters in the Standard Model and necessary facts about what the laws of nature allow. The necessitarian view of laws of nature is articulated in bold terms by David Armstrong and Fred Dretske, who argue that laws of nature are necessary relations between universals. Other anti-reductionist views of laws include those advocated by John Carroll and Marc Lange.

Laws, however, are not celebrated only by realists and metaphysicians. Carnap criticized organicist biology as pseudo-science, because it lacked laws and so the precision and quantitative prediction that he thought were essential to true science. However, he rejected the idea that laws had any modal status and thought of them

\footnotetext{
${ }^{3}$ See Ladyman [11] and the reply by Monton and van Fraassen
} 
as simply maximally general statements. Empiricists in general may prize laws for their systematizing of the phenomena but deny that they do more than describe how things behave. Kepler's laws of planetary motion, like many phenomenological laws, seem readily understood as descriptive rather than explanatory, and are not usually stated in modal form. But more fundamental laws are very often formulated modally. For example, the first law of thermodynamics (the law of conservation of energy) states that energy cannot be created or destroyed. Pauli's exclusion principle says that two electrons cannot have the same set of quantum numbers and that fermionic wavefunctions must be antisymmetrized. It is even possible to find examples of less fundamental laws that seem modally committing. In chemistry, enantiomers, i.e. stereoisomers related by a reflection, cannot be superimposed on one another. In the next section, we consider in depth the matter of the modal status of laws.

\section{2: Causation}

Scientific journals and textbooks are awash with causal claims, and much important scientific knowledge takes the form of the attribution of causal powers to objects. However, this did not stop Bertrand Russell from famously denying that science dealt with causes, and there has been a recent revival of his causal republicanism (See Price and Corry [18]). Similarly, causation was subordinate to laws in the work of logical positivists and logical empiricists, whose view of science focused on generality and universality, rather than the particular and the local. As mentioned above, the Stanford School and especially Nancy Cartwright combined their skepticism about high theory and universal laws with a robust realism about singular causation as attributed to events and processes in the laboratory. The Humean theory of causation focuses on generic causal relations between types of entities and events. In doing so, Cartwright argues, it abstracts away the complexity and specificity of concrete phenomena and misrepresents their nature. The metaphysics of causal powers that she developed is designed to draw our attention back to the way that science works in practice by attributing more or less stable causal powers to things, powers that can reinforce or inhibit each other, and whose combined action is a local outcome of singular interactions rather than the diktat of inviolable law.

Even those who do not follow Cartwright in prioritizing causal powers over lawhood are often at pains to explain how causal modality is objective albeit derivative of lawlike necessity. Armstrong, for example, argues that there is a singular causal relation between instances of properties just in case the properties in question are instantiations of universals between which there are necessary connections.

\section{3: Probability}

Probability was on Aristotle's list of modalities with good reason. Like other modal operators, such as the actuality, necessity, possibility, and potentiality operators, the operator 'probably' has the property of being non-truth-functional. Probability also seems to create intensional contexts. For instance, it was true in 2005 that it was improbable that the next president of the United States would be 
an African-American, but it was not true that it was improbable that Obama would remain an African-American. The connections between probability and potentiality are complex. On one hand, probability seems to be a kind of quantification of the potential for something to be the case. For example, there is the potential for one to be dealt four aces in a hand of five cards but not as much potential as there is to be dealt three twos. On the other hand, we use potentiality to relate finite frequencies to probabilities. Hence, after tossing a fair coin ten times and obtaining seven heads and three tails, we suppose that if we were to continue tossing the coin the relative frequencies would approach one half. While there are no actual infinite trials, we suppose that in a series of finite trials of increasing lengths the relative frequency would tend to the probability in the limit.

It is common to distinguish epistemic probabilities from objective chances. As the former may be treated in common with other epistemic modalities, it does not pose a challenge to the antirealist about objective metaphysical modality. Thus it is objective chances, or probabilities construed as real features of the world and not merely as features of our beliefs, that concern us here. David Lewis constructs a cosmology that purports to maintain objective chance despite placing very few constraints on which possible worlds are accessible from any given world. ${ }^{4}$ Other options include taking single-case chances to be a brute feature of the world, as in the theory of propensities, or somehow reducing objective chance to a result of the modal force of indeterministic laws. Prima facie the difference between a deterministic and an indeterministic theory must itself be a modal one, since any given sequence of actual events could be produced by either a deterministic or a stochastic process.

It may be that probability can be understood without metaphysical commitments to anything other than concrete events in the actual world, but it is certainly not obvious that it can be. Without question, probability is now absolutely essential to scientific theorizing and experimentation. It is of great foundational significance in statistical mechanics and quantum field theory, as well as in decoherent-histories approaches to cosmology. It is with good reason that van Fraassen calls probability the new modality of science and admits that, as an empiricist who embraces probabilism in epistemology, his own modal-frequency account of probability is what brings him closest to metaphysics.

\section{4: Equilibrium}

Equilibrium explanation is extremely important in evolutionary biology and holds great promise in the social sciences because of the development of evolutionary game theory. It is a different brand of scientific explanation than causal explanation:

Where causal explanation shows how the event to be explained was in fact produced, equilibrium explanation shows how the event

\footnotetext{
${ }^{4}$ Barry Loewer's theory of probability is a development of Lewis's.
} 
would have occurred regardless of which of a variety of causal scenarios actually transpired. (Sober [23], 201)

As with causal explanation, however, science's use of equilibrium explanation carries a prima facie commitment to the notions of physical necessity and possibility.

As an example of equilibrium explanation, Elliott Sober considers R.A. Fisher's 1931 account of why the sex ratio is 1:1 for many species. Fisher offers the explanation that if a population ever departs from this ratio, there will be a reproductive advantage favoring parental pairs that overproduce the minority sex. Supposing that males are in the majority of a given species, parental pairs that produce all female offspring will have greater fitness than pairs that produce a mix of male and female offspring, where fitness is measured in terms of number of grandchildren produced. This advantage will be retained until the species once again reaches the equilibrium sex ratio of 1:1.

It is clear that equilibrium explanation is in some sense broader than causal explanation. A causal explanation of the sex ratio for a given population would cite earlier conditions of the population, as well as evolutionary forces that move the population to the equilibrium state. The equilibrium explanation, however, need not refer to the population's earlier state, as it explains the population's equilibrium state regardless of its earlier states. Sober writes, "Whatever the actual initial sex ratio had been, the selection pressures that would have resulted would have moved the population to its equilibrium state" ([23], 202). The equilibrium explanation works for any population in equilibrium because it explains why, given any previous non-equilibrium state, the population must tend toward equilibrium. It seems then that equilibrium explanation appeals to natural necessity to account for systems being in equilibrium states.

2.2. The No-Miracles Argument. The main motivation for scientific realism in general and structural realism in particular is the no-miracles argument. The no-miracles argument is a form of inference to the best explanation. Many of those who advocate the use of IBE argue that it is only when scientific hypotheses latch onto modal structure that they are genuinely explanatory. For example, it is because the atomic structures of elements determine their possible patterns of interaction that the full valence-shell configuration of the noble gases explains why such gases tend not to participate in chemical reactions. The scientific realist argues that novel predictive success gives us a reason to believe in the unobservable entities posited by our best scientific theories. The connection between novel predictive success and unobservable entities can be understood as follows. Unobservable entities have properties and obey laws that successful theories more or less correctly describe. Hence, such theories can predict in advance what the behavior of these entities will be in novel situations and what phenomena the behavior in question will cause.

Scientific explanation creates intensional contexts without explicitly invoking either laws or causes. Like law statements and causal claims, explanations are generally not preserved under substitution of co-referential terms. For example, the temperature of the oceans is rising because of the increase in the amount of carbon dioxide in the atmosphere but not 
because of the increase in the atmosphere of the amount of the gas first isolated by Priestley. (Of course, it is only in the light of background theory that the former explanation explains anything at all). So the realist's reliance on explanation brings with it prima facie modal commitments.

It is also the realist's reliance on explanation that makes it hard to see how a Humean scientific realist can consistently motivate both of these positions. Antirealists reject the desire for explanation that motivates realism. Van Fraassen, for instance, regards all regularities in the phenomena as brute and denies that novel predictive success requires any explanation over and above the empirical adequacy of the theory in question. Since the scientific realist distinguishes herself from the antirealist in part by her commitment to explanation, the realist who repudiates causal necessity is in an awkward dialectical situation. If novelly predicted phenomena are not genuinely caused by the relevant unobservable entities, then in what sense do the latter explain the former? There are many things that are correlated with one another, but the asymmetric relation of explanation does not hold between them unless they are also causally or lawlike related. It is unclear how a realist who disavows natural necessity can make sense of the idea that unobservable entities explain the phenomena. The argument that we should believe in atoms because, if they exist, they are correlated with the phenomena is no more satisfying than the antirealist's denial that regularities ever require explanation.

\section{Against Humeanism}

Humeanism about causation is the denial that there is any such thing as causal, nomological, or natural necessity. Put another way, it is the view that there are no necessary connections in nature. For the Humean, causal connections and laws of nature are just a species of regularity among events. Of course, in everyday life and in scientific theory and practice, not all regularities are taken to be causal or lawlike. Some regularities are merely accidental patterns of correlation. Contrast, for instance, the true statement that "all the coins in the cash register are quarters," with the true statement that "every planet's orbit is an ellipse." Both statements describe regularities in nature, but only the latter is a law of nature. So statements of laws of nature are not just statements of regularities; they have some additional property, namely the property of lawlikeness. It is this property that enables statements of laws to support counterfactual conditionals and to figure in scientific explanations. The challenge for the Humean is to identify the property of lawlikeness, and so distinguish laws of nature from accidental regularities, without appealing to a notion of physical or causal necessity. If successful, the Humean can then claim that there is objective modality without incurring dubious metaphysical commitments.

3.1. The Naïve Regularity View and Epistemic Accounts of Laws. The first Humean account of laws was the naïve regularity view, which simply equated laws with universal regularities. On this view, a lawlike statement is any true universally quantified statement of the form "all $F$ s are Gs." As it denies that universal regularities must have 
some additional property in order to be laws, the view cannot distinguish laws from accidental regularities. This view was part of the early logical empiricist tradition and is now nearly universally rejected.

An early version of Humeanism about laws that does countenance a distinction between laws and accidents is the epistemic view, which characterizes the property of lawlikeness in terms of our particular epistemic attitude toward those statements that we take to be laws. Some version of the view has been defended by Strawson [24], Ayer [1], and Goodman [8]. On this view, laws are those generalizations that play a certain epistemic role: "they are believed to be true, and they are so believed because they are confirmed by their instances, and they are used in proper inductive reasoning." (Psillos [19], 141) One problem for this view is that some laws lack any positive instances. Newton's first law, for example, concerns the motion of bodies on which no forces are exerted. Since there are no such bodies, Newton's first law cannot be a law in virtue of its confirming instances. (See Armstrong [?] )

Another disadvantage of the epistemic view is that it does not explain why certain generalizations should feature in proper inductive reasoning. Since the view defines lawlike regularities in part by the role they play in induction, little in the way of explanation can be offered for why some regularities rather than others play such a role. Because the lawlikeness of a regularity depends on our epistemic attitude toward it, the view has also been criticized for the seemingly subjective and contingent nature of this criterion. It seems that we could have had different epistemic attitudes than we actually do toward any number of generalizations. Indeed, our epistemic attitude toward a specific generalization may change over time. The epistemic view has the consequences that the laws of nature change with our epistemic attitudes and that they cannot be discovered any more than customs can be. Since the idea that laws are objective, unchanging, and importantly distinct from accidental regularities is what motivated the abandonment of the naïve regularity view, the epistemic view will not detain us further.

3.2. The Objective Humean Account. The Mill-Ramsey-Lewis view, or as Psillos calls it, the web-of-laws view, is by far the most widely accepted version of Humeanism. It offers an objective characterization of lawlikeness, and so avoids the subjectivity of the epistemic view. In addition to its namesakes, the view counts Earman [6], Loewer [16], Psillos [19], and Cohen and Callender [4] among its defendants. While all but Earman identify themselves as scientific realists, we show the view to be plagued by several problems, each of which makes it difficult to reconcile with the spirit of realism.

On the MRL view, laws of nature are those regularities that feature as axioms or theorems in the best axiomatic deductive system that describes our universe. If there is one such system that can be said to offer a true description of our world, then there are many such systems. To avoid the problem of arbitrariness, Ramsey [20] and Lewis [14] both suggest that the best deductive system should be the one that strikes the best balance between simplicity and strength. For Lewis, if two or more systems tie in terms of simplicity and strength, the laws of nature are to be identified with the regularities that are common to all systems. Psillos formalizes the thesis as follows: 
(W): It is a law that all $F_{\mathrm{s}}$ are $G$ s iff (i) all $F_{\mathrm{s}}$ are $G$ s, and (ii) that all $F$ s are $G \mathrm{~s}$ is an axiom or theorem in the best deductive system $\phi$ (or if there is no unique best deductive system $\phi$, it is an axiom or theorem in all deductive systems that tie in terms of simplicity and strength).

If there are no regularities common to all such systems, then the laws of nature are indeterminate. Apart from this problem of potential indeterminacy, we argue that the view is flawed in three significant ways: its notion of strength cannot be formulated non-circularly, ${ }^{5}$ it can offer no explanation of the success of inductive inference, and it is not clear that it is compatible with a satisfactory account of the laws of the special sciences.

3.2.1. The Circularity Objection. On the web-of-laws view, we look to the best deductive axiomatized descriptions of our world to tell us which regularities count as laws of nature, and it is the theoretical virtues of simplicity and strength that tell us what those systems are. Formulating a definition of a system's simplicity is not without its difficulties, but it is the problematic notion of strength that we focus on here. Intuitively, strength has something to do with how much information can be derived from the axioms. One could say that every increase in the amount of information that is derivable from a system counts as an increase in that system's strength. This will not do, however, as any attempt to precisify such a notion seems to lead to incommensurability among systems.

Let us consider ways to explicate the intuitive notion that a system's strength is related to the amount of information derivable from its axioms. A first pass would be to formulate strength in terms of the number of facts that are derivable from a system's axioms, but this is clearly unsatisfactory. Since every system will make infinitely many facts derivable, all systems will turn out to be equally strong. Another possibility is to invoke the subset relation in the definition of strength. We could say that system A is stronger than system $B$ iff the set of facts derivable from $B$ form a proper subset of the set of facts derivable from A. But this notion of strength makes systems incommensurable with one another, as there will be many pairs of systems such that no subset relation holds between their respective sets of derivable facts. Is there a way to cash out the notion of strength that does not make systems either trivially equal or incommensurable in strength?

Psillos's alternative approach (though he does not state this explicitly) ties a system's strength to how well it vindicates our intuitions about what statements count as laws. While this strategy escapes the problems associated with formalizing the notion of strength, it is not without its own methodological difficulties. Psillos wants to exclude increases in a system's extraneous information (i.e. information about accidents and coincidences) from counting as increases in the system's strength. But if this is to be a viable method, there must be some principled way to distinguish between the derivable information that bears on a system's strength and the information that does not. Call this the principle of informational relevance. The web-of-laws proponent must be able to identify such a principle if he wishes to employ strength as a determining feature of the best axiomatic

\footnotetext{
${ }^{5}$ While a number of authors have concerned themselves with problems for the MRL account stemming from the notions of simplicity and strength, we are aware of none that have specifically addressed this problem of circularity. See Cohen and Callender [4].
} 
system. Since strength determines (in part) which deductive system is the best one, and that in turn determines which regularities are laws of nature, the definition of strength cannot invoke the distinction between accidental and lawlike regularities, as that is the very distinction it is in the service of explaining.

We argue that no principle of informational relevance, and therefore no tenable definition of strength, can be formulated without presupposing an established fact of the matter about which regularities are laws of nature. Consider Reichenbach's statement that there are no gold chunks that are larger than a cubic mile. Contrast it with the statement that there are no chunks of plutonium that are larger than a cubic mile. Psillos takes it as a datum that the latter but not the former expresses a law of nature. ${ }^{6}$ Thus, the latter but not the former should be derivable from the best deductive system. If we are choosing between two (sets of) deductive systems that are the same except that one includes the former regularity as an additional axiom, clearly we should choose the system without the additional axiom. How can the web-of-laws view justify this choice? The latter system ranks higher in simplicity; but can we not also say that the former rates higher in strength? After all, the former system enables us to derive a regularity that we are not able to derive from the latter - that all gold cubes are smaller than a cubic mile. One wants to justify the choice by saying that the more complicated system is not stronger, but in order to do this a principle of informational relevance is required.

To further illustrate the problem, consider the following passage in which Psillos asserts that the system with the additional axiom is not stronger but offers nothing in the way of explanation:

One could, of course, just add all the accidental generalizations as extra axioms to the best deductive system of the world. But in doing this, one would make this system far more complicated than it should be. If, for instance, we were to add to the best system Reichenbach's regularity that all gold cubes are smaller than one cubic mile, we would detract from its simplicity without gaining in strength. ([19], 151)

Why wouldn't the resulting system be one that has gained in strength despite losing in simplicity? The only plausible reason is that the additional regularity we derive from the amended system is not a law. This response exposes the circularity inherent in Psillos's approach to explicating the notion of strength in the web-of-laws account. On this view, laws are supposed to be just those regularities that are derivable from all true deductive systems that strike a balance between simplicity and strength. But, either systems are trivially equal or incommensurable in their strength, or there must already be a fact of the matter about exactly which regularities are laws and which are accidents in order for there to be a fact of the matter about which system is stronger. Since no unproblematic notion of strength can be defined, the view's criterion of lawlikeness does not get off the ground.

\footnotetext{
${ }^{6}$ It is worth noting that, in his explanation of why this is the case, Psillos appeals to the notion of physical impossibility, which is circular for the Humean, as he explicates the notion of physical impossibility in terms of what is incompatible with the laws.
} 
3.2.2. Laws in the Special Sciences. Apart from its problem of circularity, the web-of-laws approach faces a lack of genuine naturalistic motivation. It is not clear that we have any good reason to believe that all or even most of what we now take to be laws of nature would be the sorts of things that would find a place in a deductive axiomatic description of the world. Psillos seems to think the web-of-laws view is right in line with the practice of science, though he gives no explanation for this. He claims, "The useful fiction of an ideal deductive system of the world is not very far from the practice of science as we know it, nor far from what we now take the laws of nature to be." We are not convinced.

It may be true that attempts at unification within fundamental physics can be described as attempts to unify physics within a deductive axiomatic framework. Contemporary particle physics has been unified (for the most part) within the mathematical framework of the Standard Model, which is fundamentally a collection of symmetry groups. Mathematical systems count as deductive axiomatic systems, and so the web-of-laws view is prima facie compatible with the way fundamental physics is done. However, it is not as clear that this view can extend to the laws and practices of the special sciences.

It seems that the web-of-laws view requires all non-fundamental laws of nature to be derivable from the fundamental ones and implies that all the fundamental laws will be laws of physics. One of Psillos's footnotes supports this interpretation. He finds it worth noting that, "Carnap also took the laws of nature to be whatever lawlike statements are deducible from a set of axioms that express a certain physical theory, or more generally, "the deductive system of physics"' ([19], 303). This suggests that the web-of-laws adherent expects all laws of nature that are not themselves fundamental to be derivable from an axiomatized physics.

The difficulty with this approach is that the program of reducing special sciences to physics has often been attempted though never clearly achieved. We have little positive reason to believe that the laws of special sciences are derivable from fundamental physics, and we do have reason to believe they such derivations are computationally intractable. Our view is that the laws of the special sciences allow us to make relatively accurate predictions about macroscopic systems in the world without calculating what is happening within such systems at the minute scale of particle physics. Deriving predictions about macroscopic systems from calculations made at the scale of particle physics would require far more energy than there is in the known universe. By sacrificing some level of exactness, special-science laws allow us to make predictions that would be otherwise intractable if computed using only the fundamental laws of physics.

Consider an analogy to the famous cellular automaton, Conway's Game of Life. The "game" is played on an infinite grid in which each cell can have one of two values, black or white. As in any cellular automaton, time is discrete and there are a finite number of deterministic update rules. Given any initial conditions - any starting distribution of black and white cells - the four update rules determine exactly what the grid will look like at every later point. Something that has fascinated Game of Life enthusiasts is the "emergence" of macroscopic objects and patterns that seem to follow laws of their own. These higher-level laws quantify over certain types of cell configurations that behave in orderly, uniform ways. What is particularly interesting is that the derivation of many 
of these macro laws of the Life universe from the four underlying update rules is not a computationally tractable problem. These higher-level laws allow the Life user to make calculations about the future states of the macroscopic patterns by vastly compressing the relevant data. ${ }^{7}$ It is our view that special-science laws, like the higher-level laws of the Life universe, can be understood as tools of data-compression. A more detailed account of the view can be found in "Rainforest Realism and the Unity of Science" in [12].

The important point is that, on this view, it is characteristic of special-science laws that their derivation from the underlying laws of physics requires carrying out unmanageably large computations. Even if some of what we now take to be the laws of the special sciences are, in the strict logical sense, derivable as theorems from the axioms of fundamental physics, it is not the case that we can ever know which ones these are. Thus, if the MRL view requires all non-fundamental laws to be deducible from the axioms of physics, the view has the consequence that we cannot know which purported special-science laws are genuine laws of nature - if indeed any are. While some philosophers may accept such a view, we do not consider it a viable option. Like physics, the special sciences aim and sometimes succeed at discovering laws of nature, and any naturalistically acceptable view must respect their ability to do so.

There are two other ways that the web-of-laws theorist could try to accommodate specialscience laws, though it is unclear whether either course can offer a viable account. One strategy would be to add each non-deducible special-science law to the system as an axiom. However, this choice leads back to the circularity problem, as the web-of-laws theorist has no independent way to judge which non-deducible regularities should be counted as special-science laws, as opposed to accidents. It may also be worth noting that the notion of strength gets even murkier with respect to the special sciences, since we often cannot make straightforward derivations from special-science laws.

The other possibility would be to have separate axiomatic systems for each special science. The problem with this is that it is unclear how this version of the MRL view could represent the unity of science, i.e. both the hierarchical relationships among the special sciences and the relationship that the special sciences bear to fundamental physics. The criterion that special-science laws be theorems derivable from the axioms of fundamental physics is at least able to capture a sense in which physics is fundamental, though we think it is too strong a sense. At the other end of the spectrum is the view that each science has its own disparate axiomatic system of laws. This picture is unable to reflect the fundamentality of physics at all; it seems best-coupled with Cartwright's and Dupre's views that science is essentially disunified. The proper account of laws in the special sciences ought to steer between these two extremes; it must reflect the unique relation that holds between fundamental physics and the special sciences without requiring anything so drastic as the reduction of the special sciences to physics.

3.2.3. Inductive Inference. A third major problem for the MRL view is that it cannot explain the success of inductive inference. Recall that the Humean is committed to the claim that the only restriction on possibility is logical consistency. This means that the

\footnotetext{
${ }^{7}$ For a greater discussion of the Life universe, see Dennett [5].
} 
Humean must accept that there are infinitely many worlds that look exactly like ours up to a given point and then diverge dramatically. Consider, for instance, a world in which all the regularities hold that have held so far in our world, but only until January 1, 2085. On that date, negatively charged objects stop attracting positively charged ones, salt ceases to dissolve in water, and fish fail to suffocate on land. The Humean cannot deny that this world is possible else she commit herself to some necessary connection between, e.g., negative charge and the capacity to attract positively charged things. If this world is possible, infinitely many such worlds - that follow exactly the same course as our own for a time and then diverge - are also possible. Not only are they possible, they are far more numerous than worlds in which the observed regularities are eternal. The internal observers of these divergent worlds believe, just as we do, that the regularities they have observed in the past will continue to hold in the future. But they will be wrong. The problem for the Humean is that once she is committed to the possibility of divergent worlds, she can have no reason to believe that ours is not such a world.

The difficulty that these worlds create for induction is as follows. The problem of induction is the problem of justifying the belief that the future will be like the past. It seems that we are justified in believing that the sun will rise tomorrow, that electrons will continue to repel one another next year, and that a thousand years from now it will still be the case that nothing travels faster than the speed of light. But if it is possible for a world to act just the way our does up to some future time when its electrons will fail to repel one another, then we can have no reason to believe that our world is not one such world, and so we can have no justification for the belief that electrons will always repel one another.

It is true that an external observer looking at the entire distribution of properties throughout our spacetime would be able to tell whether or not our world is one in which electrons always repel one another. But the problem of induction is not a problem for external observers looking at the totality of facts about a world. It is inherently a problem for observers who lack full epistemic access to the future.

One might object that after January 1, 2085, when electrons continue to repel one another, we can know that we are not in a divergent world where the electron-regularity stops holding on that date. Certainly this is true, but it in no way provides a satisfactory response to the problem. For there will be an infinite number of possible worlds that are identical to ours up until to some point of divergence, for every possible point of divergence - that is, for every moment in time. Some worlds will have all the same regularities that we have observed in our own up until the moments just before they end. While we can eliminate infinitely many epistemic possibilities for every point we pass in time, there will always be infinitely many more that we cannot eliminate.

The MRL account of laws is of no use in providing a Humean response to the problem of induction. Consider the only way that the view could explain how our inductive inferences are justified. The MRL view would appeal to the fact that a given regularity is an axiom or theorem in the best deductive system describing our world as the grounds for justifying our inference that the regularity will continue to hold in the future. Since Reichenbach's regularity is not found in our best deductive system, we are not justified in believing that the future will see an absence of gold chunks larger than a cubic mile. As 
the plutonium regularity presumably does follow from the best deductive system, we are justified in believing that there will never be a plutonium chunk larger than a cubic mile. Even if we put aside the circularity problem from the previous section, this response will not be satisfactory.

Given the possibility of the deviant worlds discussed above, as internal observers, we can never know which (set of) deductive system(s) best describes our world. Since it is both metaphysically and epistemically possible ${ }^{8}$ that we occupy a world in which the electronregularity has an expiration date, we cannot know what the final deductive system of our world will look like. If we are in a world in which the regularity holds eternally, then our final system will be one in which the regularity is an axiom or theorem. ${ }^{9}$ If we are in a world in which the regularity is only temporary, the final system will not include it. We cannot know which system is the accurate description of our world until all the facts about our world are in. This entails that only an external observer looking at the totality of facts about a world can be in a position to know what the final axiomatic deductive system will be; the internal observer is never in a position to know. Since internal observers can never be in a position to know whether an observed regularity will be a part of the final deductive system, their beliefs that the regularity will persist can never be justified. ${ }^{10}$

Further, the Humean can offer no explanation for the success of inductive inference beyond dumb luck. This is especially troubling when we consider just how lucky it is that we have thus far found ourselves in a world where the regularities we observe continue to hold. Based on the sheer number of worlds in which these observed regularities fail to hold at some time, it is extremely improbably that we occupy a world in which they will continue to hold. Given this fact, the Humean ought to expect inductive inference to fail, and she cannot attribute its incredible success thus far to anything more than a cosmic miracle.

From the perspective of a scientific realist, this does not sit well. One of the driving motivations behind scientific realism is the acknowledgement that there must be a nonmiraculous explanation for the success of science. The use of induction is a cornerstone of scientific theorizing and investigation. If the Humean cannot offer a non-miraculous explanation for the success of the inductive method, neither can she offer such an explanation for the success of science.

3.2.4. Ontological Troubles. We have shown that the so-called "sophisticated" version of Humeanism faces several unique problems. But there is the more general problem with Humeanism that one of its commitments - namely, the commitment to quidditism about properties - is inconsistent with the view's motivations. Humeanism is motivated in part by the empiricist attitude that we should not be committed to the existence of what we

\footnotetext{
${ }^{8}$ For the Humean.

${ }^{9}$ Again, we are disregarding the problem of distinguishing accidental regularities that hold eternally from lawlike regularities.

${ }^{10}$ Since non-Humeans about laws accept natural necessity, they are not susceptible to this problem. NonHumeans can simply deny that there are any deviant possible worlds by ascribing necessary connections to pairs or collections of properties that feature in the relevant regularities.
} 
cannot possibly observe. Since, the reasoning goes, we can only ever observe the constant conjunction between events and never the actual causal relation, we ought not have a metaphysical account of causation as anything more than constant conjunction. But in denying that there is any physical or causal necessity in the world, the Humean must deny that there is any necessary connection between what a property is and what causal relations it bears to other properties. So the Humean must maintain that properties are something over and above their causal profiles, and they are thereby committed to the view that properties have quiddities. ${ }^{11}$

A number of philosophers (e.g. Bird [2], Hawthorne [10], Shoemaker [22]) have suggested that what physical properties are is determined, at least in part, by what they do. We can call views that fit this framework dispositional accounts of property-individuation. On these views, a physical property is a collection of dispositions, understood as the potential causal relations it can bear to other physical properties. Consider the property of being negatively charged. On a dispositional view, part of what it is to be this property is to have the capacity to attract positively charged things. Thus, on a dispositional view, there is a necessary connection between being negatively charged and being able to attract things that are positively charged. Since dispositional views of properties are committed to there being non-logical necessary connections in nature, they are incompatible with Humeanism. The Humean must deny that a property's causal profile plays any role in determining its nature. Thus, she must accept that there is something else that determines a property's nature, presumably an unobservable quiddity or primitive "thisness."

Humeanism's commitment to quidditism is deeply at odds with its empiricist motivation. Whereas the Humean rejected a metaphysically realist account of causation in order to avoid commitment to unobservables, she now finds herself committed to the existence of ineffable quiddities. Michael Esfeld [7] makes a similar point, noting that although Humean metaphysics seems ontologically parsimonious at first glance, it is not parsimonious in the end.

Not only must the Humean be committed to quiddities, she must accept that there are physically indistinguishable possible worlds - in other words, that there are differences that don't make a difference. Lewis recognized that quidditism leads to the metaphysical underdetermination of theories, which is what led him to consider the thesis of Ramseyan Humility. Quidditism entails that two worlds can share all of their physical features and yet differ only in terms of their quiddities. On the view, for instance, the role that negative charge plays in our world might be played by positive charge in another world, but the two worlds will share all of the same observable features. Since there will be multiple worlds that satisfy the Ramsey sentence describing the laws of our world, we can never know which of these worlds is our own.

Whereas the motivation for Humeanism seems to begin with a desire to keep metaphysical commitments to a minimum, it is clear that this desideratum cannot be obtained through Humeanism. In denying the notion of causal or physical necessity, the Humean

\footnotetext{
${ }^{11}$ Quiddities are to properties what haecceities are to individuals.
} 
commits herself to the existence of ineffable quiddities and differences that don't make a difference - hardly a metaphysics that can be called deflationary.

3.3. Why be Humean? Even the best version of Humeanism is plagued with problems: the above arguments suggest that it cannot be formulated non-circularly, it cannot explain why inductive inference ought to be successful, and it cannot offer a satisfactory account of laws in the special sciences. So it is worth considering why exactly one might be a Humean in the first place. This question, which Tim Maudlin [17] asks in a chapter title, is one to which he finds no satisfactory answer. Maudlin characterizes Lewis's Humeanism as the conjunction of the doctrines of Separability and Physical Statism. Separability is understood as the thesis that the complete physical state of the world is determined by the intrinsic physical state of each spacetime point and the spatio-temporal relations between those points. Maudlin argues that there is no scientific motivation for Humean metaphysics and that on the contrary quantum physics strongly suggests that the world is non-separable. Consider a pair of particles in the Singlet State. If Separability held, the joint state would be determined by the intrinsic states of each of the components and their spatio-temporal relations. But since the spin of the particles is entangled, the anti-correlation between their spins in any given direction cannot be recovered from the individual spin state of each particle (the maximally mixed state). Hence, the doctrine of Separability, which Lewis's Humeanism explicitly requires, appears to be straightforwardly false.

One could, however, hold a semi-Humean view that rejects Separability but maintains Physical Statism. Physical Statism is the doctrine that all facts about a world, including modal and nomological facts, are determined by its total physical state. It has the consequence that no two worlds can have the same total physical state but differ in their laws of nature. Maudlin argues that contemporary physics also seems to give rise to counterexamples to this thesis. Consider that cosmologists regard models of General Relativity's field equations as possible ways a universe governed by GR could be. Minkowski spacetime is a model of the GR field equations (the vacuum solution) but it is not a model only of GR laws. One could postulate a world in which Special Relativity exhausted the facts about spacetime structure and yet some other theory of gravitation held, one which would still include vacuum Minkowski spacetime as a model. These two empty Minkowski universes would share their total physical states but differ in their laws of gravitation. Since Physical Statism, the doctrine at the heart of Humeanism, appears to be in direct conflict with some of the most basic assumptions of contemporary physics, it is unclear what could motivate one to be Humean.

Maudlin considers Hume's own motivations, which relied in part on the semantic thesis that any non-analytic claims that go beyond the realm of the empirically observable are meaningless. But hardly any of those who accept Humeanism also accept a verificationist theory of meaning, and even fewer accept Hume's epistemological view that every simple idea must come from a simple impression. "The semantic theory that underlies Hume's own views has been thoroughly discredited," Maudlin writes. "Why should one have 'Humean scruples' any more?" We share in his bewilderment. 


\section{Conclusion}

While modality is much discussed in metaphysics and philosophy of science, little attention has been given to the question of what sort of modal metaphysics scientific realism requires. Ontic structural realists have made a reliance on physical modality part of their view of science. Indeed, Ladyman and Ross say that ontological commitment to modal structure is what distinguishes their view from structural empiricism. Van Fraassen himself claims that what ultimately drives him to reject scientific realism is the metaphysics of modality he thinks it entails. We have delineated the modal notions in science that give realism its prima facie commitment to objective modality, and we have explored the most plausible Humean account of it. Since sophisticated Humeanism cannot offer a reasonable account of special-science laws or explain the success of inductive inference, it is deeply at odds with the spirit of scientific realism. We thus conclude that the scientific realist must embrace natural necessity.

\section{REFERENCES}

[1] Ayer, A.J. (1956) The Problem of Knowledge. London: Macmillan.

[2] Bird, A. (2007) Nature's Metaphysics. Oxford: Clarendon Press.

[3] Cartwright, N. (2007) Nature's Capacities and their Measurement. Oxford: Clarendon Press.

[4] Cohen, J, and C. Callender (2009) A Better Best System Account of Lawhood. Philosophical Studies 145: $1-34$.

[5] Dennett, D. (1991) Real Patterns. Journal of Philosophy 88(1): 27-51.

[6] Earman, J. (1984) Laws of Nature: The Empiricist Challenge, in D. M. Armstrong. ed. R. Bogdan. Dordrecht: D. Reidel Publishing Company.

[7] Esfeld, M. (2009) The Modal Nature of Structures in Ontic Structural Realism. International Studies in the Philosophy of Science 23(2): 179-94.

[8] Goodman, N. (1983) The New Riddle of Induction, in Fact, Fiction, and Forecast. Cambridge: Harvard University Press.

[9] Hardin, C.L. and A. Rosenberg. (1982) In Defense of Convergent Realism. Philosophy of Science 49(4): 604-15.

[10] Hawthorne, J. (2001) Causal Structuralism. Philosophical Perspectives 15: 361-78.

[11] Ladyman, J. (2000). What's Really Wrong with Constructive Empiricism?: Van Fraassen and the Metaphysics of Modality. The British Journal for the Philosophy of Science 51: 83756.

[12] Ladyman, J., D. Ross, J. Collier, and J. Spurrett. (2007) Every Thing Must Go. New York: Oxford University Press.

[13] Ladyman, J. (2008) Structural Realism and the Relationship between the Special Sciences and Physics. Philosophy of Science 75(5): 744-55.

[14] Lewis, D. (1994) Humean Supervenience Debugged. Mind 103(412): 473-90.

[15] Lewis, D. (2009) Ramseyan Humility, in Conceptual Analysis and Philosophical Naturalism. eds. D. Braddon-Mitchell and R. Nola. Cambridge: MIT Press.

[16] Loewer, B. (1996) Humean Supervenience. Philosophical Topics 24: 10126.

[17] Maudlin, T. (2007) Metaphysics within Physics. New York: Oxford University Press.

[18] Price, H. and R. Corry (2007) Causation, Physics, and the Constitution of Reality: Russell's Republic Revisited. Oxford University Press.

[19] Psillos, S. (2003) Causation and Explanation. McGill-Queen's University Press.

[20] Ramsey, F. (1990) Philosophical Papers. Cambridge: Cambridge University Press.

[21] Ross, D. (2009) Ontic Structural Realism and Economics. Philosophy of Science 75(5): 732-43

[22] Shoemaker, S. (1998) Causal and Metaphysical Necessity. Pacic Philosophical Quarterly 79: 59-77. 
[23] Sober, E. (1983) Equilibrium Explanation. Philosophical Studies 43(2): 201-10.

[24] Strawson, P.F. (1952) Introduction to Logical Theory. New York: Wiley. 